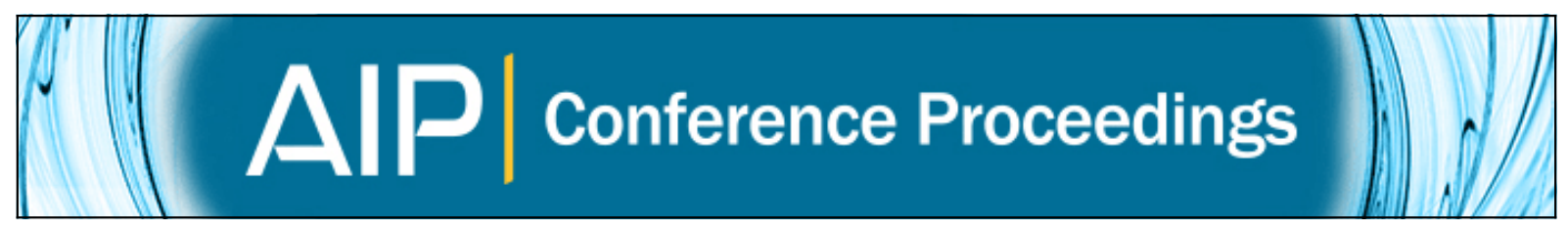

\title{
An interpolation problem arising in a coupling of the finite element method with holomorphic basis functions
}

K. Gürlebeck, U. Kähler, and D. Legatiuk

Citation: AIP Conference Proceedings 1648, 440004 (2015); doi: 10.1063/1.4912655

View online: http://dx.doi.org/10.1063/1.4912655

View Table of Contents: http://scitation.aip.org/content/aip/proceeding/aipcp/1648?ver=pdfcov

Published by the AIP Publishing

\section{Articles you may be interested in}

Convergence of the finite element method with holomorphic functions

AIP Conf. Proc. 1558, 513 (2013); 10.1063/1.4825540

On a special finite element based on holomorphic functions

AIP Conf. Proc. 1479, 308 (2012); 10.1063/1.4756124

Coupling finite element and boundary element methods on a mixed solid-fluid/fluid-fluid boundary for radiation or scattering problems

J. Acoust. Soc. Am. 96, 3792 (1994); 10.1121/1.410568

Analysis of finite-element matrices arising from discretizations of helicity functionals J. Appl. Phys. 67, 5815 (1990); 10.1063/1.345973

A finite element method with local trigonometric basis for close coupling equations

J. Chem. Phys. 74, 6133 (1981); 10.1063/1.441003 


\title{
An Interpolation Problem Arising in a Coupling of the Finite Element Method with Holomorphic Basis Functions
}

\author{
K. Gürlebeck*, U. Kähler ${ }^{\dagger}$ and D. Legatiuk** \\ ${ }^{*}$ Chair of Applied Mathematics, Bauhaus-Universität Weimar, Germany \\ ${ }^{\dagger}$ CIDMA - Center for R \& D in Mathematics and Applications, Universidade de Aveiro, Portugal \\ ${ }^{* *}$ Research Training Group 1462, Bauhaus-Universität Weimar, Germany
}

\begin{abstract}
The purpose of this paper is to prove an interpolation theorem which arises in a method of coupling of a finite element and an analytical solution for boundary value problems with singularities.
\end{abstract}

Keywords: FEM, coupling, interpolation, holomorphic functions

PACS: 02.10.Ud, 02.10.Yn, 02.60.Ed, 02.70.Dh.

\section{INTRODUCTION}

In previous research $[2,3]$ the main idea of a new method of coupling an analytical and a numerical solution (FEsolution) for boundary value problems with a singularity has been introduced. This method allows to get a continuous coupling between analytical and finite element solutions through the whole interaction interface. For that purpose a special element that contains an exact solution to the differential equation with the correct singularity and socalled coupling elements was constructed. The request for these coupling elements is to insure $C^{0}$ continuity for displacements, instead of coupling only via nodes between two solutions as in usual approaches. For that reason a special interpolation operator has been constructed that preserves the analytical solution on the coupling interface, couples it continuously with coupling elements which have a polynomial connection to the standard elements. In [1] following P. G. Ciarlet [4] some basic steps for the convergence analysis of the proposed method have been performed. In this theory one of the most important roles is played by the unisolvence property of the interpolation operator that is used for the finite element approximation. In [5] it was proved that the interpolation problem is uniquely solvable for an arbitrary number of nodes. In [6] it was shown, that a coupling error has a significant influence on the quality of results. To estimate this coupling error one needs to work with a modified interpolation function, therefore it has to be proven that this function is still the solution of the original interpolation problem.

\section{INTERPOLATION THEOREM}

Let us consider the following interpolation function in polar coordinates on the disk of radius $r_{\mathrm{A}}$ (for all details we refer to [5])

$$
\begin{aligned}
f_{n}(\varphi)= & \sum_{k=0,2}^{N_{1}} r_{\mathrm{A}}^{\frac{k}{2}}\left[a_{k}\left(\kappa e^{i \varphi \frac{k}{2}}+e^{-i \varphi \frac{k}{2}}\right)+\frac{k}{2} \bar{a}_{k}\left(e^{-i \varphi \frac{k}{2}}-e^{-i \varphi\left(\frac{k}{2}-2\right)}\right)\right]+ \\
& +\sum_{k=1,3}^{N_{2}} r_{\mathrm{A}}^{\frac{k}{2}}\left[a_{k}\left(\kappa e^{i \varphi \frac{k}{2}}-e^{-i \varphi \frac{k}{2}}\right)+\frac{k}{2} \bar{a}_{k}\left(e^{-i \varphi \frac{k}{2}}-e^{-i \varphi\left(\frac{k}{2}-2\right)}\right)\right],
\end{aligned}
$$

where a number of the basis functions is related to a number $n$ of interpolation nodes $\varphi_{n}$ as follows:

$$
N_{1}=n-2^{m}, \quad \text { where }\left\{\begin{array} { l l } 
{ m = 1 } & { \text { for even } n , } \\
{ m = 0 } & { \text { for odd } n , }
\end{array} \quad N _ { 2 } = n - 2 ^ { m } , \text { where } \left\{\begin{array}{ll}
m=0 & \text { for even } n, \\
m=1 & \text { for odd } n .
\end{array}\right.\right.
$$

Theorem 1. For given $n$ different arbitrary interpolation nodes $\varphi_{j} \in[-\pi, \pi]$ and $n$ arbitrary complex numbers $y_{j}$ exists a unique function (1), such that

$$
f_{n}\left(\varphi_{j}\right)=y_{j}, \quad j=0, \ldots, n-1 .
$$


Proof. At first, we rewrite the interpolation function (1) as follows

$$
f_{n}(\varphi)=\sum_{\substack{k=-N_{1}, \ldots \\-N_{1}+2, \ldots}}^{N_{1}} A_{k} e^{i \varphi \frac{k}{2}}+\sum_{\substack{k=-N_{2}, \ldots \\-N_{2}+2, \ldots}}^{N_{2}} B_{k} e^{i \varphi \frac{k}{2}}
$$

where the coefficients $A_{k}$ and $B_{k}$ are related to the original coefficients $a_{k}$ and $\bar{a}_{k}$ by the following equations

$$
A_{k}=\left\{\begin{array}{cc}
r_{\mathrm{A}}^{\frac{|k|}{2}} a_{|k|}+\frac{|k|}{2} r_{\mathrm{A}}^{\frac{|k|}{2}} \bar{a}_{|k|}, & \text { for }-N_{1} \leq k<-N_{1}+4, \\
r_{\mathrm{A}}^{\frac{|k|}{2}} a_{|k|}+\frac{|k|}{2} r_{\mathrm{A}}^{\frac{|k|}{2}} \bar{a}_{|k|}-\frac{|k|+4}{2} r_{\mathrm{A}}^{\frac{|k|+4}{2}} \bar{a}_{|k|+4}, & \text { for }-N_{1}+4 \leq k \leq-2, \\
a_{0}(\kappa+1)-2 r_{\mathrm{A}}^{2} \bar{a}_{4}, & \text { for } k=0, \\
\kappa r_{\mathrm{A}} a_{2}-r_{\mathrm{A}} \bar{a}_{2}, & \text { for } k=2, \\
r_{\mathrm{A}}^{\frac{k}{2}} \kappa a_{k}, & \text { for } 4 \leq k \leq N_{1},
\end{array}\right.
$$

and

$$
B_{k}=\left\{\begin{array}{cc}
-r_{\mathrm{A}}^{\frac{|k|}{2}} a_{|k|}+\frac{|k|}{2} r_{\mathrm{A}}^{\frac{|k|}{2}} \bar{a}_{|k|}, & \text { for }-N_{2} \leq k<-N_{2}+4, \\
-r_{\mathrm{A}}^{\frac{|k|}{2}} a_{|k|}+\frac{|k|}{2} r_{\mathrm{A}}^{\frac{|k|}{2}} \bar{a}_{|k|}-\frac{|k|+4}{2} r_{\mathrm{A}}^{\frac{|k|+4}{2}} \bar{a}_{|k|+4}, & \text { for }-N_{2}+4 \leq k \leq-1, \\
\kappa r_{\mathrm{A}}^{\frac{1}{2}} a_{1}-\frac{3}{2} r_{\mathrm{A}}^{\frac{3}{2}} \bar{a}_{3}, & \text { for } k=1, \\
\kappa r_{\mathrm{A}}^{\frac{3}{2}} a_{3}-\frac{1}{2} r_{\mathrm{A}}^{\frac{1}{2}} \bar{a}_{1}, & \text { for } k=3, \\
\kappa r_{\mathrm{A}}^{\frac{k}{2}} a_{k}, & \text { for } 5 \leq k \leq N_{2} .
\end{array}\right.
$$

An interpolation function (3) contains $2 n-1$ coefficients, but we have only $n$ interpolation nodes, therefore we have to find $n-1$ additional relations which should help us to identify uniquely the coefficients $A_{k}$ and $B_{k}$. By studying the equations (4)-(5) one can observe that the coefficients $A_{-|k|}$ and $B_{-|k|}$ could be expressed in terms of the coefficients $A_{|k|}$ and $B_{|k|}$, respectively. Thus, we get the following equations

$$
A_{-|k|}=\left\{\begin{array}{cc}
\kappa^{-1} A_{|k|}+\frac{|k|}{2} \kappa^{-1} \bar{A}_{|k|}, & \text { for }-N_{1} \leq k<-N_{1}+4 \\
\kappa^{-1} A_{|k|}+\frac{|k|}{2} \kappa^{-1} \bar{A}_{|k|}-\frac{|k|+4}{2} \kappa^{-1} \bar{A}_{|k|+4}, & \text { for }-N_{1}+4 \leq k \leq-4 \\
(\kappa-1)^{-1}\left[A_{2}+\kappa^{-1} \bar{A}_{2}\right]+(\kappa-1)^{-1}\left[\bar{A}_{2}+\kappa^{-1} A_{2}\right]-3 \kappa^{-1} \bar{A}_{6}, & \text { for } k=-2
\end{array}\right.
$$

and

$$
B_{-|k|}=\left\{\begin{array}{cc}
-\kappa^{-1} B_{|k|}+\frac{|k|}{2} \kappa^{-1} \bar{B}_{|k|}, & \text { for }-N_{2} \leq k<-5, \\
-r_{\mathrm{A}}^{\frac{3}{2}}\left(\kappa B_{3}+\frac{1}{2} \bar{B}_{1}\right)\left(\kappa^{2} r_{\mathrm{A}}^{\frac{3}{2}}-\frac{3}{4} r_{\mathrm{A}}^{\frac{3}{2}}\right)^{-1}+ & \\
+\frac{3}{2} r_{\mathrm{A}}^{\frac{3}{2}}\left(\kappa \bar{B}_{3}+\frac{1}{2} B_{1}\right)\left(\kappa^{2} r_{\mathrm{A}}^{\frac{3}{2}}-\frac{3}{4} r_{\mathrm{A}}^{\frac{3}{2}}\right)^{-1}-\frac{7}{2} \kappa^{-1} \bar{B}_{7}, & \text { for } k=-3,
\end{array}\right.
$$




$$
B_{-|k|}=\left\{\begin{array}{l}
-\frac{1}{2} \kappa^{-1} B_{1}-\frac{3}{2} \kappa^{-1} r_{\mathrm{A}}^{\frac{3}{2}}\left(\kappa \bar{B}_{3}+\frac{1}{2} B_{1}\right)\left(\kappa^{2} r_{\mathrm{A}}^{\frac{3}{2}}-\frac{3}{4} r_{\mathrm{A}}^{\frac{3}{2}}\right)^{-1}+ \\
+\frac{3}{4} r_{\mathrm{A}}^{\frac{3}{2}}\left(\kappa B_{3}+\frac{1}{2} \bar{B}_{1}\right)\left(\kappa^{2} r_{\mathrm{A}}^{\frac{3}{2}}-\frac{3}{4} r_{\mathrm{A}}^{\frac{3}{2}}\right)^{-1}-\frac{5}{2} \kappa^{-1} \bar{B}_{5}, \quad \text { for } k=-1 .
\end{array}\right.
$$

Finally we combine two sums in (3) into the one sum

$$
f_{n}(\varphi)=\sum_{k=0}^{n-1} C_{k} e^{i \varphi \frac{k}{2}}
$$

with the coefficients $C_{k}$ are defined by

$$
C_{k}= \begin{cases}A_{k}, & \text { if } k \text { is even, } \\ B_{k}, & \text { if } k \text { is odd. }\end{cases}
$$

Thus we have obtained an equivalent interpolation problem

$$
\sum_{k=0}^{n-1} C_{k} e^{i \varphi_{j} \frac{k}{2}}=y_{j}, \quad j=0, \ldots, n-1,
$$

with exactly $n$ unknown coefficients $C_{k}$. The matrix of the equivalent interpolation problem is a Vandermonde matrix, and its determinant is given by

$$
\operatorname{det}(\ldots)=\prod_{1 \leq j<k \leq n}\left(e^{i \frac{1}{2} \varphi_{k}}-e^{i \frac{1}{2} \varphi_{j}}\right) \neq 0 .
$$

The product in this determinant is not equal to zero due to the assumption on the interpolation nodes. Thus the interpolation problem (10) is uniquely solvable for an arbitrary number of nodes, and for an arbitrary right hand side, i.e. we can uniquely identify the coefficients $C_{k}$, and consequently $A_{k}$ and $B_{k}$ for all values of $k$.

The next step in this proof is to show that from the coefficients $A_{k}$ and $B_{k}$ we can uniquely calculate the original coefficients $a_{k}$ and $\bar{a}_{k}$. For simplicity we will consider a transformation from $a_{k}, \bar{a}_{k}$ to $A_{k}, B_{k}$ which is given by (4)(5), and we will show that the corresponding transformation matrix has non-zero determinant. The dimension of the corresponding transformation matrix depends on even or odd number of interpolation nodes. Here we will consider the case of an odd number of nodes, and the case of an even number can be proved analogously. Let us write the equations (4)-(5) in a matrix form

$$
\left(\begin{array}{c}
A_{-N_{1}} \\
B_{-N_{2}} \\
\vdots \\
B_{N_{2}} \\
A_{N_{1}}
\end{array}\right)=\left(\begin{array}{ccccc}
\frac{N_{1}}{2} r_{\mathrm{A}}^{\frac{N_{1}}{2}} & 0 & \ldots & 0 & r_{\mathrm{A}}^{\frac{N_{1}}{2}} \\
0 & \frac{N_{2}}{2} r_{\mathrm{A}}^{\frac{N_{2}}{2}} & \ldots & -r_{\mathrm{A}}^{\frac{N_{2}}{2}} & 0 \\
\vdots & \vdots & \ldots & \vdots & \vdots \\
0 & 0 & \ldots & \kappa r_{\mathrm{A}}^{\frac{N_{2}}{2}} & 0 \\
0 & 0 & \ldots & 0 & \kappa r_{\mathrm{A}}^{\frac{N_{1}}{2}}
\end{array}\right)\left(\begin{array}{c}
\bar{a}_{N_{1}} \\
\bar{a}_{N_{2}} \\
\vdots \\
a_{N_{2}} \\
a_{N_{1}}
\end{array}\right) .
$$

In sequel we will denote by $M$ the transformation matrix, and for the case of an odd number of interpolation nodes $\operatorname{dim}(M)=(2 n-1) \times(2 n-1)$. For forthcoming discussion it's important to underline a block $M_{7}$ of the matrix $M$, which is located in the middle $(i, j=n-3, \ldots, n+3)$

$$
M_{7}=\left(\begin{array}{ccccccc}
\frac{3}{2} r_{\mathrm{A}}^{\frac{3}{2}} & 0 & 0 & 0 & 0 & 0 & -r_{\mathrm{A}}^{\frac{3}{2}} \\
0 & r_{\mathrm{A}} & 0 & 0 & 0 & r_{\mathrm{A}} & 0 \\
0 & 0 & \frac{1}{2} r_{\mathrm{A}}^{\frac{1}{2}} & 0 & -r_{\mathrm{A}}^{\frac{1}{2}} & 0 & 0 \\
0 & 0 & 0 & \kappa+1 & 0 & 0 & 0 \\
-\frac{3}{2} r_{\mathrm{A}}^{\frac{3}{2}} & 0 & 0 & 0 & \kappa r_{\mathrm{A}}^{\frac{1}{2}} & 0 & 0 \\
0 & -r_{\mathrm{A}} & 0 & 0 & 0 & \kappa r_{\mathrm{A}} & 0 \\
0 & 0 & -\frac{1}{2} r_{\mathrm{A}}^{\frac{1}{2}} & 0 & 0 & 0 & \kappa r_{\mathrm{A}}^{\frac{3}{2}}
\end{array}\right) .
$$


Now we will calculate the determinant of $M$. Decomposing $\operatorname{det}(M)$ by the rows $2 n-1, \ldots, n+4$, and then by rows $1, \ldots, n-4$ we obtain

$$
\operatorname{det}(M)=\left(\prod_{i=1}^{n-4} M_{i i}\right) \cdot\left(\prod_{i=n+4}^{2 n-1} M_{i i}\right) \cdot\left|M_{7}\right|,
$$

where the matrix $M_{7}$ is given by (11). The determinant of this matrix can be explicitly calculated, and we get

$$
\left|M_{7}\right|=\frac{3}{4} r_{\mathrm{A}}^{6}(\kappa+1)^{3}(\kappa-1) .
$$

Finally we obtain the following expression for the determinant

$$
\operatorname{det}(M)=\frac{3}{4} r_{\mathrm{A}}^{6}(\kappa+1)^{3}(\kappa-1) \prod_{i \in \Lambda} M_{i i}, \quad \Lambda=\{1, \ldots, n-4, n+4, \ldots, 2 n-1\} .
$$

Looking on the structure of the transformation equations (4)-(5) we see, that the entries $M_{i j}$, and particularly $M_{i i}$, depend only on parameters $N_{1}, N_{2}, r_{\mathrm{A}}$, and $\kappa$, which are all positive by their definition. Therefore the product in (12) cannot be equal to zero. The remaining part is to discuss the determinant $\left|M_{7}\right|$, which contains $(\kappa+1)^{3}(\kappa-1)$. The parameter $\kappa$ is a material constant, and by assumptions on the material properties we know that $\kappa \in(1,3)$. Therefore

$$
(\kappa+1)^{3}(\kappa-1) \neq 0 .
$$

Thus we have shown, that the determinant $\operatorname{det}(M)$ is not equal to zero, and therefore the transformation matrix has an inverse for an odd number of nodes. The case of an even number of nodes is analogous.

Thus we have proved that the interpolation problem (2) is uniquely solvable for an arbitrary number of interpolation nodes and for an arbitrary right hand side.

In [5] we have already proved that the interpolation problem (2) is uniquely solvable for an arbitrary number of nodes. The new proof is needed for future study on convergence and stability of the proposed method. Because it's easier to get estimates working with a Vandermonde matrix and with the transformation matrix $M$. We would only like to mention the fact that the coefficients $a_{k}$ and $\bar{a}_{k}$ are connected by the complex conjugation. In the presented proof we assume, for sake of shorter explanations, that they all are different. But for practical calculations we have to decompose them into real and imaginary parts, and in this case all steps of the presented proof are anyway going straight forward.

\section{ACKNOWLEDGMENTS}

The research of the third author is supported by the German Research Foundation (DFG) via Research Training Group "Evaluation of Coupled Numerical Partial Models in Structural Engineering (GRK 1462)", which is gratefully acknowledged. The research of the second author is supported by Portuguese funds through the CIDMA - Center for Research and Development in Mathematics and Applications, and the Portuguese Foundation for Science and Technology ("FCT-Fundação para a Ciência e a Tecnologia”), within project PEst-OE/MAT/UI4106/2014.

\section{REFERENCES}

1. S. Bock, K. Gürlebeck, D. Legatiuk, Convergence of the finite element method with holomorphic functions. AIP Conference proceedings 1558, 513 (2013).

2. S. Bock, K. Gürlebeck, D. Legatiuk, On a special finite element based on holomorphic functions, AIP Conference proceedings 1479, 308 (2012).

3. S. Bock, K. Gürlebeck, D. Legatiuk, On the continuous coupling between analytical and finite element solutions, Le Hung Son $\&$ Wolfgang Tutschke eds. Interactions between real and complex analysis, pp. 3 - 19. Science and Technics Publishing House, Hanoi, 2012.

4. Philippe G. Ciarlet, The finite element method for elliptic problems, North-Holland Publishing Company, 1978.

5. Klaus Gürlebeck and Dmitrii Legatiuk, On the continuous coupling of finite elements with holomorphic basis functions. Hypercomplex Analysis: New perspectives and applications, ISBN 978-3-319-08770-2, Birkhäuser, Basel.

6. Dmitrii Legatiuk and Hung Manh Nguyen, Improved convergence results for the finite element method with holomorphic functions. Submitted. 